\title{
O que se "ensina" no Ensino Superior: avaliando conhecimentos, competências, valores e atitudes
}

\author{
- Diana Dias * \\ - Diana Soares ** \\ - Claisy Marinho-Araújo *** \\ - Leandro Silva Almeida *****
}

\section{Resumo}

A educação superior contemporânea contempla a promoção de valores cívicos e éticos que visam à transformação da sociedade. Esta missão implica não só veicular competências técnico-científicas, como promover competências transversais, fundamentais nos vários domínios de atuação profissional. Cada vez mais, a tendência para a conjugação de conhecimentos e competências, a par de valores e atitudes sociais, cívicas e éticas se concretiza nos currículos e nas práticas pedagógicas. Dentro de uma metodologia qualitativa, com recurso a análise de conteúdo, este estudo explora os perfis de competências apresentados pelas Instituições de Educação Superior em Portugal, considerando um conjunto de cursos clássicos na área da engenharia, mais concretamente Engenharia Civil, Engenharia Eletrotécnica e Engenharia Mecânica de todo o país. Os resultados destacam a primazia dos conhecimentos e competências técnico-científicas. O desenvolvimento cognitivo do estudante continua a ser mais valorizado, pese embora a tendência, ainda que tênue, de uma crescente valorização das atitudes e dos valores cívicos enquanto produto de um Ensino Superior de qualidade.

Palavras-chave: Ensino superior. Competências. Conhecimentos. Atitudes e valores.

Doutora em Ciências da Educação e licenciada em Psicologia pela Universidade do Porto, é Agregada à Universidade do Minho. Vice-Reitora e Professora Associada da Universidade Europeia I Laureate International Universities. Investigadora integrada do Centro de Investigação em Políticas do Ensino Superior; E-mail: diana.dias@universidadeeuropeia.pt.

** Doutora em Psicologia da Educação pela Universidade do Minho. Professora auxiliar convidada na Universidade Europeia Laureate International Universities; investigadora integrada do Centro de Investigação em Políticas do Ensino Superior. Email: diana.soares@universidadeeuropeia.pt.

*** Doutora em Psicologia pela Universidade de Brasília, pós-doutorado e estágio sênior pela Universidade do Minho. Professora e pesquisadora do Instituto de Psicologia e do Programa de Pós-Graduação em Processos de Desenvolvimento Humano e Saúde da Universidade de Brasília; E-mail: claisy@unb.br.

**** Doutor em Psicologia pela Universidade do Porto. Professor Catedrático de Psicologia da Educação no Instituto de Educação da Universidade do Minho. Investigador do Centro de Investigação em Educação (CIEd) na Universidade do Minho; E-mail: leandro@ie.uminho.pt. 


\section{Introdução}

A complexidade e imprevisibilidade da vida econômica e social dos nossos dias requerem um esforço permanente de atualizações de políticas e práticas dos sistemas educativos. As exigências sociais e configurações sócio-políticas contemporâneas instalam a urgência por uma educação superior (ES) que considere, na construção do perfil profissional dos estudantes, tanto os embasamentos técnico, científico, conceitual e metodológico quanto um conjunto ampliado de competências transversais (MARINHOARAUJO; ALMEIDA, 2016). Nessa direção, pesquisas e políticas públicas sinalizam alternativas de formação na ES, por meio do desenvolvimento de competências, que oportunizem estudo e aprimoramento permanentes, agilidade, criatividade, independência e postura inquisitiva para tomada de decisão (ANANIADOU; CLARO, 2009; SOARES et al., 2017). Na mesma linha, relevam-se competências como o trabalho em equipe, a preocupação com questões éticas e morais e a aprendizagem autônoma enquanto dimensões-chave na formação dos estudantes de ES (DIAS; SOARES, 2016). Progressivamente assume-se que a construção de um perfil profissional integra, juntamente com competências e conhecimentos técnicos específicos, as dimensões inerentes ao próprio desenvolvimento intra e interpessoal do estudante, a sua cultura e seu posicionamento cívico em face de problemáticas sociais (MARINHO-ARAUJO; ALMEIDA, 2016; SHEPHARD et al., 2015; SOARES; DIAS, 2016). Neste quadro, a formação superior pode entender-se como um processo multidimensional, ancorado no desenvolvimento de múltiplas competências, que decorre do entrelaçamento dinâmico entre a história de vida do sujeito, sua trajetória de formação, saberes das experiências, relações de trabalho, reconhecimento social da profissão e as peculiaridades do momento histórico-cultural (BERTOLIN, 2017).

Diante da diversidade de tais contribuições, irá eleger-se, nesse artigo, a interface entre o desenvolvimento de competências nos cursos de graduação e a construção do perfil profissional dos estudantes. Acredita-se que a investigação em torno da formação acadêmica dos estudantes e a construção dos seus perfis profissionais possam fundamentar análises que subsidiem políticas institucionais voltadas à melhoria da qualidade na ES, em um esforço de aproximação da academia ao mundo do trabalho (WERQUIN, 2012). O contexto da pesquisa relatada no presente trabalho é o ES de Portugal, focando os resultados de aprendizagem (learning outcomes) que as instituições dizem promover nos seus estudantes. Entendendo-se os resultados de aprendizagem 
enquanto afirmações do que se espera que o estudante adquira e demonstre no final de determinada qualificação (ADAM, 2004), este estudo pretende analisar que conhecimentos, competências, atitudes e valores são valorizados nos programas curriculares do ES português, particularizando uma amostra de cursos de engenharia.

\section{O conceito de resultados de aprendizagem}

O conceito de learning outcome (resultado de aprendizagem - RA), seguindo a definição proposta por Adam (2004; 2008), remete para o que um estudante deve saber, compreender e/ou ser capaz de demonstrar no final de um dado período de aprendizagem. Assume-se, assim, como um instrumento fiável para a descrição clara e operacional, não só do que é esperado que os estudantes venham a saber, compreender e fazer com base em uma determinada qualificação, mas também como podem transitar de uma qualificação para outra, dentro de um dado sistema de ensino e entre os diversos países europeus (mobilidade e transparência). Para além dessa descrição, os resultados de aprendizagem devem referir as metodologias e os critérios segundo os quais os estudantes serão avaliados (MOON, 2004).

Desta forma, a tônica é colocada na coerência dos resultados de aprendizagem inerentes a uma determinada qualificação, em função do que é esperado que o estudante adquira em termos de conhecimentos e competências, em função da área de estudos e da unidade curricular correspondente. Esta abordagem do processo ensinoaprendizagem permite aos estudantes conhecerem, de antemão, o que é esperado que saibam, compreendam e sejam capazes, quer em uma dada unidade curricular, quer em um dado ciclo de estudos (ADAM, 2004; SCOTT, 2011). Como tal, a implementação de RA nos currículos implica uma perspectiva da educação centrada no estudante ("studentcentered learning"), por oposição ao foco no professor (WRIGHT, 2011). A ênfase passa a ser a aprendizagem (em detrimento do ensino) e os outputs que dela advêm (SCOTT, 2011). Esta visão da ES potencializa o desenvolvimento de competências autorregulatórias por parte dos estudantes, sugerindo o seu impacto na qualidade das aprendizagens (ALMEIDA, 2002; PINTRICH, 2000; SCHUNK; ZIMMERMAN, 2008).

Tipologias de RA

Sendo inegável a relevância do conceito de RA no âmbito da educação superior, as questões relativas à sua natureza e dimensões que lhe estão inerentes permanecem em 
aberto. Os diversos modelos propostos na literatura nem sempre convergem entre si, o que torna a tipificação dos RA um processo ambíguo e pouco definido (AAMODT; HOVDHAUGEN, 2008; ADAM, 2008; DIAS; SOARES, 2017; SCOTT, 2011). Assim, se por um lado, os quadros nacionais e europeus das qualificações diferenciam os RA em conhecimentos, atitudes, aptidões/habilidades e competências (CHAKROUN, 2010; WINTERTON; DELAMARE-LE DEIST; STRINGFELLOW, 2006), outros modelos enfatizam o desenvolvimento de conhecimentos, as aptidões cognitivas e intelectuais, as aptidões genéricas e as aptidões práticas como dimensões-chave dos RA (MOON, 2004). Acrescentando alguma complexidade ao tema, a própria definição de competência é, em si, mesma, alvo de múltiplas interpretações (MARTíN; GONZÁLEZ; GONZÁLEZ, 2017). Entende-se, assim, que as competências se associem, intimamente, às operações cognitivas, seja, por exemplo, a capacidade de abstrair conhecimento, ou a sua aplicação em outros contextos (RANGEL; MARCARZEL; PIMENTA, 2016).

Partindo dos vários posicionamentos teóricos, Nusche (2008) apresenta uma tipologia de RA para o ES, baseada na separação entre RA cognitivos e não-cognitivos (Figura 1). Reconhece-se, no entanto, que qualquer categorização dos RA envolve necessariamente alguma artificialidade, uma vez que os diferentes componentes da aprendizagem são interdependentes e, muitas vezes, sobrepostos (BOWEN, 1977). Assim, esta tipologia (tal como qualquer outra) deve ser compreendida com significado heurístico, com o objetivo de identificar as diferentes dimensões da aprendizagem e respectivas metodologias de avaliação.

Figura 1 - Tipologia de RA

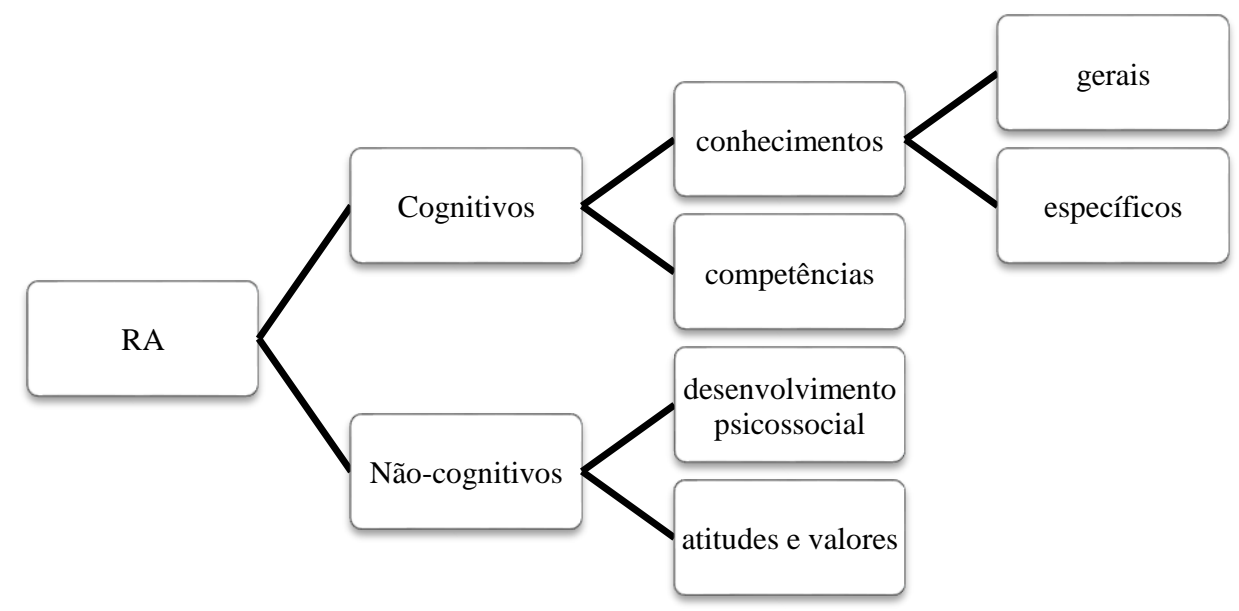

Fonte: NUSCHE (2008). 
A dimensão cognitiva dos RA remete para a construção de conhecimentos e o desenvolvimento de capacidades intelectuais e competências (STRIKE; POSNER, 1992). Em termos gerais, os RA de base cognitiva vão desde os mais específicos domínios do conhecimento, até aos processos mais globais de raciocínio e da resolução de problemas (SHAVELSON; HUANG, 2003). Na literatura, é possível encontrar diversas classificações de RA de base cognitiva por exemplo, (LIM et al., 2007) algumas inspiradas na taxonomia de objetivos educacionais proposta por Bloom (1956). Esta taxonomia fornece uma leitura dos objetivos que a educação pretende desenvolver, indo além do conhecimento factual e da compreensão, para incluir competências mais relacionadas com a resolução de problemas, como a aplicação, a síntese, a análise e a avaliação.

Os RA de base cognitiva podem diferenciar-se em termos de conhecimento e competências (NUSCHE, 2008). O conhecimento reporta-se a ideias, materiais ou fenômenos, sendo esse conhecimento reconhecido através da evocação ou do reconhecimento (BLOOM, 1956) podendo focalizar-se no conteúdo geral ou no domínio específico. O conhecimento de um conteúdo geral refere-se a um curriculum básico, considerado como aprendizagem essencial (MAEROFF, 2006), que segue a linha de um conjunto de conhecimentos gerais ou básicos, em termos culturais, que qualquer estudante do ES deveria adquirir independentemente da sua área de estudos (KILGO; EZELL SHEETS; PASCARELLA, 2015). O conhecimento de um domínio específico, por sua vez, refere-se ao conhecimento apreendido em uma área científica em particular, tal como a Sociologia ou a Arquitetura, o que remete para um conhecimento com características mais técnicas e científicas.

Por sua vez, as competências cognitivas são baseadas em processos complexos de pensamento, tais como os raciocínios verbal e quantitativo, o processamento de informação, a compreensão, as operações analíticas, o pensamento crítico, a resolução de problemas e a avaliação de ideias novas (NUSCHE, 2008). A característica comum dos RA em termos de competências cognitivas é que vão além das áreas específicas de estudo, sendo transferíveis entre diferentes campos científicos e permitindo aos estudantes operar em diversos contextos (PASCARELLA; TERENZINI, 2005), aproximando-se do conceito de competências transversais e genéricas (MARINHO-ARAÚJO; RABELO, 2015).

Os RA de base não cognitiva reportam-se a mudanças nas crenças ou ao desenvolvimento de certos valores (NUSCHE, 2008). As instituições de ES incluem, 
frequentemente, na sua missão, referência ao desenvolvimento de dimensões nãocognitivas, assumindo que o seu papel vai além da promoção da aquisição de conhecimentos e competências operacionais (DIAS; SOARES, 2016). Os RA não cognitivos podem ser desenvolvidos tanto em contexto de sala de aula, como em atividades extracurriculares promovidas para complementar as curriculares, tais como a tutoria, o aconselhamento, o esporte ou mesmo as atividades em associações e centro acadêmicos. A promoção de tais atividades acaba por ser um indicador da valorização das aprendizagens não-cognitivas, enquanto formação complementar a que ocorre em sala de aula.

Diversos autores se debruçam sobre as (possíveis) taxonomias teóricas e empíricas de RA não cognitivos. Pascarella e Terenzini (2005) compilaram mais de 2.500 estudos, no contexto americano, sobre o impacto do ES nos seus alunos. Entre as variáveis mais frequentemente focadas encontram-se os RA relacionados com o desenvolvimento psicossocial, incluindo aspectos do autodesenvolvimento, tais como o desenvolvimento da identidade e da autoestima, ou o desenvolvimento relacional dos estudantes relativamente a pares, docentes, instituição e mesmo condições de vida. Nusche (2008), corroborando Pascarella e Terenzini (2005), sugere a subdivisão dos RA não-cognitivos em desenvolvimento psicossocial, atitudes e valores. A primeira dimensão remete para o desenvolvimento da identidade e das perceções acerca de si (como autoestima, autoconceito e autoeficácia), a par do relacionamento interpessoal. As atitudes e valores englobam, por sua vez, questões de cidadania, responsabilidade social e moral, princípios éticos e deontológicos e o respeito pela diversidade cultural.

Em síntese, o presente estudo parte da tipologia proposta por Nusche (2008), para diferenciar RA de base cognitiva e não-cognitiva, visando analisar que conhecimentos, atitudes e valores e competências as instituições de ES Portuguesas enfatizam nos seus programas curriculares em cursos de engenharia.

\section{Métodos}

Medidas

O processo de acreditação dos ciclos de estudo no ES, em Portugal, inicia-se com o preenchimento de um Guião de Avaliação, por parte das IES, que é, posteriormente, proposto à Agência Portuguesa de Avaliação e Acreditação e Avaliação da Educação 
Superior - A3ES. No formulário de acreditação é solicitada a descrição dos resultados de aprendizagem a adquirir pelos estudantes no âmbito do ciclo de estudos em causa.

Para este estudo, consideraram-se os resultados de aprendizagem mencionados em uma amostra representativa dos programas de engenharia clássica (ou seja, Engenharia Civil, Engenharia Eletrotécnica e Engenharia Mecânica). Os dados foram cedidos pela A3ES, salvaguardando-se a confidencialidade das instituições avaliadas. Analisaram-se todos os pedidos de Acreditação de programas de Engenharia propostos à A3ES, entre 2009 e 2014, o que corresponde a um total de dezessete documentos. Ambos os subsistemas (Universitário e Politécnico) e setores (público e privado) que constituem o ES português, estão representados. As propostas de acreditação referem-se à criação de primeiros e segundos ciclos, em três modalidades distintas: 1을 Ciclo (Licenciatura) e 2응 Ciclo (Mestrado Integrado e Mestrado Independente). A Licenciatura corresponde a três anos iniciais de educação superior. Os mestrados correspondem a mais dois anos de formação superior. No caso de um curso com mestrado integrado, a duração da formação é de cinco anos (sem pausa e sem mudança de curso nem instituição). Nos mestrados independentes, não há esse seguimento, podendo ocorrer mudança de área científica e de instituição de ES.

Dos dezessete relatórios analisados, dez referem-se a mestrados independentes, quatro a licenciaturas e os restantes três a mestrados integrados. A maioria dos documentos analisados pertence a instituições públicas (10). A distribuição em relação ao subsistema de ensino é mais equilibrada, havendo igual número de instituições universitárias e politécnicas.

Procedimentos de análise de dados

Tendo em consideração o objetivo deste estudo e a natureza dos dados, optou-se por uma metodologia de investigação qualitativa. Concretamente, os dados foram alvo de uma análise de conteúdo, utilizando o software MAXQDA, versão 12. Este software foi escolhido pela sua versatilidade e flexibilidade para incluir a orientação metodológica adotada para esta pesquisa.

Usando como referência metodológica a abordagem de Mendes et al. (2017), adotou-se um processo de categorização, sobretudo dedutivo. Partiu-se do quadro 
teórico proposto por Nusche (2008), assumindo-se como categorias de análise os RA cognitivos (conhecimentos gerais e específicos, e competências gerais e específicas) e RA não-cognitivos (desenvolvimento psicossocial, atitudes e valores). As transcrições foram codificadas de acordo com este conjunto de temas e foram analisadas utilizando uma abordagem de comparação constante. Os dados foram codificados por parágrafo e frase. Como atributos foram consideradas variáveis relativas a: (i) subsistema de ensino (Universitário e Politécnico), setor (público e privado) e graus propostos (Licenciatura, Mestrado Integrado e Mestrado Independente).

\section{Resultados}

Em um primeiro momento e considerando a abordagem preliminar da análise de conteúdo dos documentos em estudo, procedeu-se a uma análise das frequências de palavras que constam dos textos codificados (Tabela 1).

Tabela 1 - Descrição das palavras mais frequentes no excerto codificado dos relatórios

\begin{tabular}{lcc}
\hline \hline Palavra & Frequência & Percentual \\
\hline Competências & 27 & 2,15 \\
Capacidade & 23 & 1,83 \\
Conhecimentos & 22 & 1,75 \\
Gestão & 20 & 1,59 \\
Civil & 18 & 1,43 \\
Engenharia & 17 & 1,35 \\
Sistemas & 15 & 1,19 \\
Desenvolver & 14 & 1,11 \\
Problemas & 13 & 1,03 \\
Mestre & 12 & 0,95 \\
Público & 11 & 0,87 \\
Resolução & 10 & 0,8 \\
Soluções & 10 & 0,8 \\
\hline \hline
\end{tabular}

Fonte: Os autores (2018).

Verifica-se que a palavra mais mencionada $(n=27)$ é "competências", logo seguida das palavras "capacidade" $(n=23)$ e conhecimentos $(n=22)$. Destacam-se, ainda, as palavras "gestão" ( $n=20)$, “civil” ( $n=18)$, "engenharia" ( $n=17)$, e "sistemas" ( $n=15)$. Com uma frequência próxima deste segundo grupo de palavras, aparecem duas novas 
palavras: "desenvolver" ( $n=14)$ e "problemas" $(n=13)$. Os termos referidos correspondem a $13,4 \%$ do total de palavras.

Apresentam-se, em seguida, os resultados que decorrem da análise de conteúdo. Em uma primeira fase, descrevem-se os resultados considerando a totalidade das respostas. No segundo momento, diferenciam-se os resultados em função do setor (público vs. privado), subsistema (universitário vs. politécnico) e ciclo de estudos (Licenciatura, Mestrado Integrado e Mestrado Independente).

Quando considerada a totalidade das respostas, verifica-se que se codificaram cerca de 140 extratos narrativos, o que, em média, corresponde a 8,24 referências por documento, ou seja, em média, cada Pedido de Acreditação apresenta cerca de oito referências a diferentes RA.

No que respeita às categorias RA, conforme indicado no Gráfico 1, verifica-se que a maioria é de base cognitiva $(77,9 \%)$ e, dentro destes, $78 \%$ correspondem a competências (e apenas um quinto se reporta a conhecimentos). Dentro das competências e dos conhecimentos elencados, predominam os específicos ( 87,5 dos conhecimentos e $56,7 \%$ das competências referidas são específicos). No caso dos RA de base não cognitiva (22\% do total de RAs codificados); a percentagem de referências às atitudes e valores e ao desenvolvimento psicossocial é equilibrada (55\% e 45\%, respetivamente).

Gráfico 1 - Percentagem de cada tipologia de RA para a totalidade dos dados

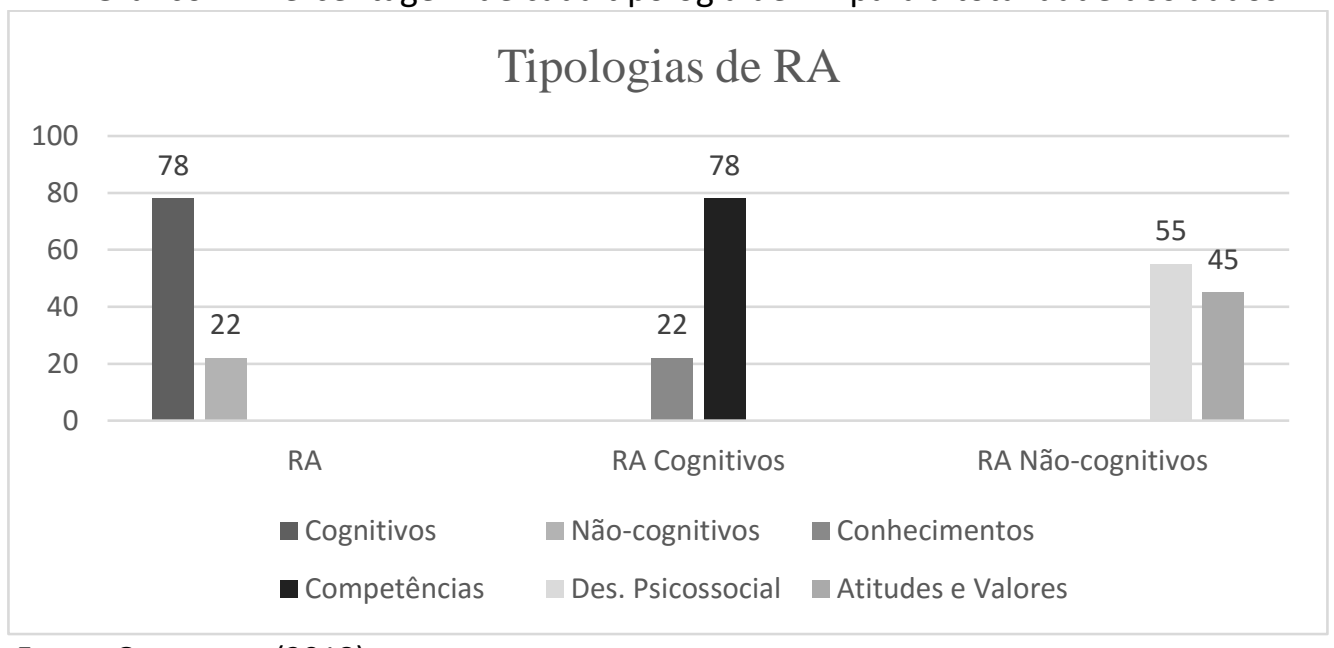

Fonte: Os autores (2018).

Posteriormente, cruzando as categorias de RA e setor de ES, verifica-se que as propostas provenientes do ensino público têm, em média, 10,2 RA por documento, enquanto o setor privado tem uma média de sete. Focando os RA de base cognitiva, verifica-se que é o setor público que apresenta uma média superior (8,1 RA por documento), em relação ao setor 
privado (média de 6,7). Da mesma forma, a média do ensino público continua superior no que diz respeito a RA relacionados com competências de base cognitiva $(5,4$ para uma média de 3,3 no setor privado); no entanto, o mesmo não se verifica a RA referentes a conhecimentos, apresentando aqui o ensino privado uma média superior (1,7 para 1,4 no ensino público). Ainda no que respeita a competências, verifica-se que o ensino privado tem uma média superior de referências de competências específicas (3,3 para 2,7 do público), o que contrasta com o fato de não haver qualquer codificação de competências gerais para este setor de ensino, enquanto no ensino público há uma média de 2,6 em competências gerais.

O mesmo padrão ocorre ao nível dos conhecimentos. As instituições de ensino privado não referem qualquer RA relativo a conhecimentos gerais (contra uma média de 0,2 no ensino público), mas apresentam uma média superior de referências a conhecimentos específicos $(1,7)$ face ao ensino público $(1,1)$. Em relação aos RA de base não cognitiva é o ensino público que apresenta médias superiores (2,1 em face de 0,3 do privado). De referir ainda que, se no ensino público os associados ao desenvolvimento psicossocial são referidos, em média, uma vez por relatório, não ocorrendo qualquer referência no ensino privado. No que diz respeito às atitudes e valores, é também o ensino público que apresenta médias superiores $(1,1)$, em relação ao ensino privado $(0,3)$.

Focando, agora, os subsistemas de ensino, verifica-se que o ensino universitário apresenta uma média ligeiramente superior de referências a RA $(8,6)$, em relação ao ensino politécnico $(8,0)$. As universidades referem mais RA de base cognitiva (média de 6.9, por comparação com a média 6,1 do ensino politécnico). Dentro dos RA cognitivos, é o ensino politécnico que apresenta uma média superior de referência a competências (5,3 em contraste com a média de 4,6 do universitário), invertendo-se esta tendência no caso dos RA relacionados com conhecimentos (ensino universitário apresenta uma média 2,3, face à média de 0.8 do ensino politécnico). Uma análise detalhada das competências evidencia que as competências gerais são, em média, mais referidas pelo ensino universitário (2,9 em face de 1,7 no politécnico), enquanto as competências específicas são mais referidas pelo ensino politécnico $(3,6)$ em relação ao universitário $(1,7)$. No caso dos RA relacionados com conhecimentos, é o ensino universitário que apresenta uma média superior, seja nos conhecimentos específicos (1,9 para 0,8$)$, seja nos conhecimentos gerais $(0,4)$, não havendo qualquer referência a estes no ensino politécnico. Por outro lado, quando analisados os RA de base não-cognitiva, verifica-se que o ensino politécnico apresenta uma média ligeiramente 
superior (1,9 contra 1,7). Esta diferença de médias fica a dever-se essencialmente aos RA relativos ao desenvolvimento psicossocial, em que a média do politécnico $(0,9)$ é superior à média do universitário $(0,7)$, havendo médias idênticas nos dois subsistemas para atitudes e valores.

Por último, a diferenciação das categorias de RA em função do ciclo de estudos evidencia que os mestrados independentes apresentam uma média superior $(8,8)$ de referência de RA, quando comparada com a dos mestrados integrados $(8,3)$ e das licenciaturas $(6,8)$. Focando os RA de base cognitiva, a mesma distribuição se mantém, com os mestrados independentes a apresentarem uma média de 6,9, os mestrados integrados de 6,7 e as licenciaturas de 5,0. Relativamente às competências, a tendência mantém-se: os mestrados independentes apresentam uma média superior $(5,4)$, seguidos dos mestrados integrados $(4,7)$ e das licenciaturas $(4,3)$. Contudo, verifica-se que são os mestrados integrados que apresentam uma média superior relativamente a competências gerais $(3,3)$, comparando com as licenciaturas e mestrados independentes (2,0 e 1,9 respectivamente). No que diz respeito a competências específicas, os mestrados independentes voltam a tomar a preponderância com uma média de 3,5, para uma média de 2,3 nos primeiros ciclos, e 1,3 nos mestrados integrados. Por outro lado, no que se refere a RA relacionados com conhecimentos, são os mestrados integrados que assumem a predominância com uma média de RA deste tipo por relatório ( 1,5 nos mestrados independentes e 0,8 nas licenciaturas). Os RA de conhecimento geral são apenas referidos nos mestrados independentes $(0,3)$. Por sua vez, os mestrados integrados apresentam uma média bastante superior $(2,0)$ de conhecimentos específicos, comparativamente com os mestrados independentes $(1,2)$ e com as licenciaturas $(0,8)$. Em relação a RA de base não cognitiva, os mestrados independentes apresentam uma média ligeiramente superior $(1,9)$ aos restantes. Estes valores distintos devem-se essencialmente aos RA relacionados com o desenvolvimento psicossocial (médias de 0,9 por documento para mestrados independentes, 0,8 nas licenciaturas e 0,7 para mestrados integrados), já que cada um dos graus refere igualmente RA relativo a atitudes e valores $(1,0)$.

\section{Discussão}

Este estudo teve como principal objetivo analisar quais conhecimentos, competências, atitudes e valores as instituições de ES portuguesas valorizam enquanto 
RA adquirido pelos seus estudantes no âmbito da sua formação, focando, particularmente, a área científica das engenharias clássicas. Esta análise permite, ainda, conhecer como se operacionalizam os RA, diferenciando os setores público e privado, os subsistemas universitário e politécnico, e os ciclos de ensino (1.ํ ciclo, mestrado independente e mestrado integrado).

Em termos gerais, os resultados apontam para a implementação efetiva dos RA nos currículos de engenharia. A análise das palavras mais frequentes evidencia a relevância das "competências", "capacidade" e "conhecimentos" enquanto elementos cruciais na elaboração dos resultados de aprendizagem. As palavras "gestão", "civil", "engenharia" e "sistemas" são também bastante referenciadas, remetendo já para especificidades da área científica em estudo (engenharia). Por fim, "desenvolver" e "problemas", termos bastante mencionados, remetem novamente para as dimensõesbase dos resultados de aprendizagem. Como tal, entendendo os RA como postulados sobre o que um aluno deve saber, compreender e/ou ser capaz de demonstrar no final de um período de aprendizagem (ADAM, 2004; 2008; SCOTT, 2011), a frequência das palavras usadas parece um bom indicador da implementação dos RA na ES.

Analisando o conteúdo dos documentos, verifica-se um nítido esforço para operacionalizar e concretizar os RA na oferta formativa e no currículo da ES. Atendendo aos resultados obtidos, a função principal dos RA parece ser mais informativa, enfatizando as competências e conhecimentos que o estudante deverá deter no final da sua formação. Com efeito, os dados apontam para a predominância de competências de base cognitiva em detrimento dos fatores não-cognitivos. Todavia, apesar desta diferenciação, certo é que as atitudes e valores e o desenvolvimento psicossocial (RA nãocognitivos) vão assumindo uma crescente relevância, particularmente notória no ensino público. No que respeita à diferenciação entre conhecimentos específicos e competências cognitivas, verifica-se que os primeiros são mais frequentemente referidos por instituições de ES privadas (sobretudo conhecimentos específicos) e por universidades (sobretudo conhecimentos gerais). As competências cognitivas, por outro lado, são mais referidas pelas instituições de ES públicas e por politécnicos (sobretudo ao nível das competências específicas). 
Então, assim sendo, o que é que as Instituições de ES dizem que ensinam? A resposta terá de salientar especialmente a multiplicidade e abrangência de conhecimentos, competências gerais e específicas, valores, atitudes e fatores associados ao desenvolvimento psicossocial dos estudantes que se supõem que aprendam. Se por um lado, é notório o predomínio das dimensões cognitivas, certo é que as não-cognitivas parecem adquirir uma preponderância crescente, muito em resposta às exigências sociais e culturais que hoje são colocadas às instituições de ES e aos seus graduados. Neste sentido, a ES assume, como sua missão, não só a formação técnica e científica dos seus estudantes, como também o seu desenvolvimento cívico e cultural.

O delineamento do perfil do profissional do século XXI exige, como tal, o acompanhamento de sua construção por mecanismos desenvolvimentais complexos, que articulem as características pessoais e as especificidades profissionais, competências cognitivas e não-cognitivas às novas condições do mercado global. Neste sentido, os RA surgem como indicadores dos conhecimentos, competências, atitudes e valores que as instituições de ES esperam imprimir nos seus estudantes e futuros profissionais. Acreditase, por fim, que os percursos acadêmicos dos estudantes, considerando os RA como indicadores dos seus perfis profissionais, possam constituir-se uma oportunidade de aproximação entre academia e mercado de trabalho.

\section{Conclusão}

As transformações sociais desafiam as instituições de ensino superior a mudanças na sua missão e práticas pedagógicas. É importante analisar como os conhecimentos, competências, atitudes e valores desenvolvidos pelos diplomados servem sociedades pautadas por crescente complexidade e incerteza. A investigação tem mostrado essa preocupação por parte dos governos e das instituições, concluindo-se neste artigo que, mesmos em cursos clássicos, se observa essa maior consciência da explicitação necessária dos resultados de aprendizagem a assegurar pelos diplomados e da sua atualização face aos desafios da sociedade.

Neste sentido, analisando os descritivos dos cursos por parte dos responsáveis pedagógicos, na elaboração dos resultados de aprendizagem encontramos alusão significativa a termos como "competências", "capacidades" e "conhecimentos". Esta 
ocorrência está em sintonia com as orientações pedagógicas da "Declaração de Bolonha", destacando um processo de ensino-aprendizagem organizado em torno do desenvolvimento de competências e onde o estudante assume um papel relevante. Ou seja, a oferta formativa das Engenharias mais clássicas parece estar alinhada com as premissas subjacentes à efetiva implementação dos RA nos seus currículos. Aliás, a frequência relativa a termos como "desenvolver" e "problemas" são exemplos paradigmáticos das dimensões-base dos resultados de aprendizagem e alinham-se com os principais pressupostos do que deve ser um resultado de aprendizagem bem formulado (DIAS, 2018). Tal como foi já referido, denota-se na construção dos resultados de aprendizagem dos diferentes cursos analisados uma demanda para os operacionalizar, tornando-os mais informativos na descrição concreta das competências e conhecimentos que o estudante deverá possuir no final da sua formação.

Este estudo permite concluir, ainda, que os RA se reportam essencialmente à componente científico-técnica dos cursos, ou seja, a competências cognitivas. Neste sentido, estão menos presentes as competências ditas transversais, mais reportadas às atitudes, valores e às formas de pensar e de agir em contextos de mudança tecnológica e social. No entanto, é o setor público que se destaca pela progressiva relevância que dá aos resultados de aprendizagem de caráter menos cognitivo. Por sua vez, as instituições de ensino superior privadas que se centram mais nos conhecimentos específicos, em detrimento das competências gerais, situam-se no subsistema universitário. É possível que, centrando-se este artigo em cursos nas áreas clássicas de engenharia, este enfoque cognitivo possa estar mais representado por comparação com outras áreas científicas no campo das ciências e das humanidades. Por outro lado, alguma diferenciação encontrada nos resultados obtidos, mesmo tratando-se de cursos clássicos de uma única área científica, reforça que estas análises devem sempre atender aos contextos identitários das instituições e à natureza dos cursos.

Em síntese, inseridas em um contexto social particularmente exigente, as instituições de ES debatem a necessidade de atender a um equílibrio nem sempre fácil de obter, entre o desenvolvimento cognitivo dos seus estudantes e o seu desenvolvimento psicossocial. Hoje em dia, não basta saber ou saber fazer; torna-se necessário também saber ser e saber estar. Para além da formação científica e/ou profissionalizante dos seus públicos, a 
ES acresce à sua missão o desenvolvimento cívico e cultural dos seus graduados. Neste contexto, os RA assumem-se como indicadores privilegiados das tendências de objetivos esperados no final de uma graduação, sejam estas conhecimentos, competências, atitudes e/ou valores cívicos e morais. Por último, partindo dos contributos deste estudo, importaria, assim, em uma tentativa de melhor compreender o cenário da ES, alargar esta análise a outras áreas científicas, permitindo obter respostas mais amplas e compreensivas face ao que é valorizado, "ensinado" e, efetivamente "aprendido" pelas instituições e pelos graduados, fornecendo novos insights para a discussão dos designs curriculares e dos processos que sustentam a elaboração e formulação de RA no âmbito da ES. 


\section{Referências}

AAMODT, P. O.; HOVDHAUGEN, E. Assessing higher education learning outcomes as a result of institutional and individual characteristics. IMHE: Programme on Institutional Management in Higher Education, Paris, 2008.

ADAM, S. Using learning outcomes: a consideration of the nature, role, application and implications for European education of employing "learning outcomes" at the local, national and international levels. In: UNITED KINGDOM BOLOGNA SEMINAR, 2004, Edimburgo. Anais... Edimburgo: Heriot-Watt University, 2004. p. 30.

ADAM, S. Learning Outcomes based Higher Education: the scottish experiences. In: PAPER PRESENTED TO THE BOLOGNA SEMINAR. 2008. Edimburgo. Anais... Edimburgo: Heriot-Watt University, 2008.

ALMEIDA, L. S. Facilitar a aprendizagem: ajudar os alunos a aprender. Psicologia Escolar e Educacional, [S.I.], v. 6, p. 155-165, Campinas: ABRAPEE, 2002.

ANANIADOU, K.; CLARO, M. 21st century skills and competences for new milleninum learners in OECD countries. Paris: [s.n.], 2009.

BERTOLIN, J.; BERTOLIN, J. A formação integral na educação superior e o desenvolvimento dos países. Cadernos de Pesquisa, São Paulo, v. 47, n. 165, p. 848-871, set. 2017.

BLOOM, B. Taxonomy of educational objectives: the cognitive domain. New York: Longman, 1956.

BOWEN, H. Investment in learning: the individual and social value of American higher education. San Francisco: Jossey-Bass, 1977.

CHAKROUN, B. National qualification frameworks: from policy borrowing to policy learning. European Journal of Education, [S.I.], v. 45, n. 2, p. 199-216, 2010.

DIAS, D. Learning Outcomes in European Higher Education. In: PATHAK, T. P.; MILLER, S. J. (Ed.). Encyclopedia of international higher education systems and institutions. Dordrecht: Springer Netherlands, 2018.

DIAS, D.; SOARES, D. Retrieving civic dimensions in higher education curricula: is learning improving students' civic development?. In: EDULEARN16 PROCCEDINGS, 16., 2016. Barcelona. Anais... Barcelona: IATED DIGITAL LIBRARY, 2016. Disponível em: <http://library.iated.org/view/DIAS2016RET>. Acesso em: 21 fev. 2018.

DIAS, D.; SOARES, D. Learning outcomes in higher education: designing a conceptual map for portuguese academia. In: INTED2017, 17., 2017 Valencia. Anais...Valencia: IATED, 2017. Disponível em: <http://library.iated.org/view/DIAS2017LEA>. Acesso em: 21 fev. 2018. 
KILGO, C. A.; EZELL SHEETS, J. K.; PASCARELLA, E. T. The link between high-impact practices and student learning: some longitudinal evidence. Higher Education, [S.I.], v. 69, p. 509-525, 2015.

LIM, D. H. et al. Typology of learning outcomes in cognitive domain: what is said vs. what is measured. In: INTERNATIONAL RESEARCH CONFERENCE IN THE AMERICAS OF THE ACADEMY OF HUMAN RESOURCE DEVELOPMENT, 2007. Indianapolis. Anais... Indianapolis: IRCTA, 2007.

MAEROFF, G. Grading higher education, beyond the rankings: measuring learning in higher education. Columbia: Hechinger Institute on Education and the Media; Columbia University, 2006.

MARINHO-ARAUJO, C. M.; ALMEIDA, L. S. Abordagem de competências, desenvolvimento humano e educação superior. Psicologia: Teoria e Pesquisa, Brasília, v. 32, n. esp, p. 1-10, 2016.

MARINHO-ARAÚJO, C.; RABELO, M. L. Avaliação educacional: a abordagem por competências. Avaliação, Sorocaba, SP, v. 20, n. 2, p. 443-466, 2015.

MARTÍN, S. C.; GONZÁLEZ, M. C.; GONZÁLEZ, S. S. Evaluación de competencias: Retos en la formación práctica de los pedagogos. Meta: Avaliação, Rio de Janeiro, v. 9, n. 25, p. 90-109, 2017.

MENDES, R. M. et al. A análise de conteúdo como uma metodologia. Cadernos de Pesquisa, São Paulo, v. 47, n. 165, p. 1044-1066, set. 2017.

MOON, J. Linking evels, learning outcomes and assessment criteria. Paper presented at the Seminar "Using Learning Outocomes. In: PAPER PRESENTED TO THE BOLOGNA SEMINAR. 2004. Edimburgo. Anais... Edimburgo: Heriot-Watt University, 2004.

NUSCHE, D. Assessment of learning outcomes in higher education. OECD Education Working Papers, [S.I.], n. 15, 2008.

PASCARELLA, E. T.; TERENZINI, P. T. How college affects students: a third decade of research. San Francisco, CA: Jossey-Bass, 2005.

PINTRICH, P. R. The role of goal orientation in self-regulated learning. In: BOEKAERTS, M.; PINTRICH, P.; ZEIDNER, M. (Ed.). Handbook of self-regulation: theory, research and applications. San Diego, CA: Academic Press, 2000.

RANGEL, M.; MARCARZEL, M. S.; PIMENTA, M. F. A Trajetória das competências e habilidades em educação no Brasil: das avaliações em larga escala para as salas de aula. Meta: Avaliação, Rio de Janeiro, v. 8, n. 22, p. 28-47, 2016.

SCHUNK, D. H.; ZIMMERMAN, B. J. Motivation and self-regulated learning: theory, research, and application. New York: Routledge, 2008. 
SCOTT, I. The learning ooutcome in higher education: time to think again?. Worcester Journal of Learning and Teaching, [S.I.], n. 5, p. 1-8, 2011.

SHAVELSON, R. J.; HUANG, L. Responding responsibly to the frenzy to assess learning in higher education. Change, [S.I.], v. 31, p. 10-19, 2003.

SHEPHARD, K. et al. Seeking learning outcomes appropriate for "education for sustainable development" and for higher education. Assessment \& Evaluation in Higher Education, [S.I.], v. 40, n. 6, p. 855-866, 18 ago. 2015.

SOARES, D. et al. More competent and more equal: higher education as a supporter of soft skills development. . In: INTED2017, 17., 2017 Valencia. Anais...Valencia: IATED, 2017. Disponível em: <http://library.iated.org/view/SOARES2017MOR>. Acesso em: 21 fev. 2018.

SOARES, D.; DIAS, D. Understanding lifelong learning through the eyes of Portuguese higher education institutions. In: EDULEARN16 PROCCEDINGS, 16., 2016. parcelona. Anais... Barcelona: IATED DIGITAL LIBRARY, 2016. Disponível em: <http://library.iated.org/view/SOARES2016UND>. Acesso em: 21 fev. 2018.

STRIKE, K. A.; POSNER, G. A revisionist theory of conceptual change. In: DUSCHL, R. A.; HAMILTON, R. J. (Ed.). Philosophy of science, cognitive psychology, and educational theory and practice. Albany: Suny Press, 1992.

WERQUIN, P. The missing link to connect education and employment: recognition of non-formal and informal learning outcomes. Journal of Education and Work, Reino Unido, v. 25, n. 3, p. 259-278, 2012.

WINTERTON, J.; DELAMARE-LE DEIST, F.; STRINGFELLOW, E. Typology of knowledge, skills and competences. [s.I: s.n.], 2006.

WRIGHT, G. B. Student-centered learning in Higher Education. International Journal of Teaching and Learning in Higher Education, [S.I.], v. 23, n. 3, p. 92-97, 2011.

Recebido em: 21/02/2018

Aceito para publicação em: 12/07/2018 


\title{
What is "taught" in Higher Education: Assessing knowledge, skills, values and attitudes
}

\begin{abstract}
In current times, higher education contemplates the promotion of civic and ethical values, aiming the transformation of society. This mission involves not only technical and scientific competences but also transversal competences, fundamental in all professional activity fields. Increasingly, the tendency to combine knowledge and skills, along with social, civic, and ethical values and attitudes, is embodied in curricula and pedagogical practices. In a qualitative methodology using content analysis, this study explores the competency profiles presented by Higher Education Institutions in Portugal. The results highlight the primacy of technical-scientific knowledge and specific skills. The student's cognitive development is still more valued, despite the tendency, albeit tenuous, for a growing appreciation of civic attitudes and values as a product of a quality higher education.
\end{abstract}

Keywords: Higher Education. Competences. Knowledge. Aptitudes and values.

\section{¿Qué se "enseña" en la Enseñanza Superior? Evaluando conocimientos, competencias, valores y actitudes}

\section{Resumen}

La Educación Superior contemporánea abarca la promoción de valores cívicos y éticos que conducen a transformar la sociedad. Esta misión conlleva no sólo divulgar competencias técnico-científicas sino también promover competencias transversales, fundamentales en los diversos dominios de actuación profesional. Cada vez más la tendencia hacia la conjugación de conocimientos y competencias, junto a valores y actitudes sociales, cívicas y éticas se concreta en los planes de estudios y en las prácticas pedagógicas. Bajo un análisis cualitativo, este estudio explora los perfiles de competencias presentados por las Instituciones de Educación Superior de Portugal, específicamente en el área de Ingeniería Civil, Ingeniería Electrónica e Ingeniería Mecánica. Los resultados destacan la primacía de los conocimientos y competencias técnico-científicas. El desarrollo cognitivo del estudiante sigue siendo más valorado, pese a la tendencia, aunque tenue, de una 
creciente valoración de las actitudes y de los valores cívicos como producto de una Enseñanza Superior de calidad.

Palabras clave: Enseñanza Superior. Competencias. Conocimientos. Actitudes y valores. 